\title{
La preocupación por la calidad de la educación y su valor social
}

\author{
The concern about educational quality and its social value
}

\author{
Felipe Martínez-Rizo \\ Universidad Autónoma de Aguascalientes, México
}

Recibido: 15/10/2018

Aceptado: 19/12/2018

\begin{abstract}
The article is based on the observation that in many countries there is widespread concern about the quality of their respective education systems. Next, the support of this concern is analyzed; at least in part, it is based on a superficial interpretation of the results of large-scale performance tests, such as those promoted by the OECD, which are known by the acronym PISA. Then we reflect on the risk that a limited vision of the notion of educational quality can lead to neglect fundamental dimensions of the educational endeavor, and we conclude with considerations about the social value of education.

KEY WORDS: Educational quality; Evaluation; Social value of education.

\section{RESUMEN}

El artículo parte de la constatación de que en muchos países se detecta una extendida preocupación por la calidad de sus respectivos sistemas educativos. En seguida se analiza el sustento que tiene esa preocupación, en parte basada en una interpretación superficial de los resultados de pruebas en gran escala de rendimiento, como las promovidas por la OCDE que se conocen con la sigla PISA. Luego se reflexiona sobre el riesgo de que una visión limitada de la noción de calidad educativa puede llevar a descuidar dimensiones fundamentales de la tarea educativa, y se concluye con consideraciones sobre el valor social de la educación.
\end{abstract}

PALABRAS CLAVE: Calidad de la educación; evaluación; valor social de la educación. 


\section{La preocupación por la calidad de la educación antes y ahora}

La manera en que los actores involucrados en cuestiones educativas conciben la calidad de dichas actividades se ha transformado sustancialmente en las dos últimas décadas, y esa transformación ha ido acompañada de un crecimiento sin precedentes en la amplitud y la profundidad de la preocupación al respecto.

Para entender cabalmente la importancia del cambio ocurrido, conviene recordar que los sistemas educativos modernos se desarrollaron a lo largo del siglo XIX y durante el XX, con un avance paulatino y muy desigual entre países en lo que se refiere a la proporción de niños y jóvenes que asistía a los diversos grados, en relación con el total de quienes estaban en edad de hacerlo.

En los países que se industrializaron tempranamente -el Reino Unido, Francia, Prusia y luego Alemania, otros países europeos y los Estados Unidos-el proceso ocurrió primero, y poco a poco se extendió otras regiones, incluyendo colonias de las potencias europeas, los países de América Latina ya independientes y, con sus propias modalidades, Japón.

Hasta fines del siglo XX ni siquiera se contaba con estadísticas fiables con las que se pudiera comparar con precisión el avance de cada país en lo relativo a acceso y permanencia de niños y jóvenes en la escuela, mucho menos había forma de comparar el nivel de preparación que alcanzaban los jóvenes que terminaban los diversos niveles escolares en cada país. En cada país había mecanismos para cuidar la calidad educativa, que incluían requisitos para que una persona pudiera dedicarse a la docencia, y sistemas de supervisión (inspectorados). Los países más importantes se enorgullecían de sus respectivos sistemas educativos, y las autoridades correspondientes no echaban de menos la ausencia de mecanismos que les permitieran valorar los niveles de aprendizaje alcanzados.

Lo que cambió tal estado de cosas fue el desarrollo de herramientas capaces de medir en forma aparentemente objetiva los niveles de aprendizaje: la psicometría que, a partir de las ideas de pioneros europeos como Binet, surgió en los Estados Unidos, durante la primera mitad del siglo XX.

Los estudiosos del tema consideran que para 1950 el proceso había llegado a una razonable madurez, reflejada en la versión clásica de la teoría de las pruebas (Classical Test Theory), plasmada por Harold Gulliksen (Theory of Mental Tests), siguiendo las huellas de Edward Thorndike; de Lewis Terman, que adaptó las ideas de Binet en Stanford; de la prueba de aptitud académica del College Board (Scholastic Aptitude Test); y del Educational Testing Service de Princeton. (De Landsheere, 1996; Martínez Arias, 1995)

En el contexto de la Guerra Fría, el lanzamiento por la Unión Soviética del primer satélite artificial -el Sputnik, en octubre de 1957- hizo que en los Estados Unidos se produjera una reacción de gran inquietud ante el riesgo de que el país se viera superado por su rival en la carrera espacial y armamentista. Se coincidía en la necesidad de tener más y mejores científicos e ingenieros, y todo eso llevó a que surgiera una fuerte preocupación por la calidad de la educación, en especial en las áreas de matemáticas y ciencias naturales. (Mathison y Ross, 2008)

En ese contexto se comprende el creciente interés por las pruebas de rendimiento en gran escala, que en las décadas siguientes se concretó en la implantación, con carácter obligatorio, por casi todos los estados americanos, de pruebas basadas en estándares mínimos de desempeño; en la creación de las pruebas nacionales del National Assessment of Education Progress; en el movimiento de estándares educativos; en la meta fijada por los gobernadores de los 50 estados de que en el año 2000 los alumnos del país serían los primeros del mundo; y en la obligación, para todos los estados, de aplicar anualmente pruebas censales en grados clave de la enseñanza elemental y media, que estableció en 2002 la ley No Child Left Behind. (Baker y Choppin, 1990; Mathison y Ross, 2008)

Con pocas excepciones, como el Reino Unido, Australia y los Países Bajos, hasta fines del siglo XX en otros países el uso de pruebas estandarizadas distaba mucho de tener la importancia que se le daba en Estados Unidos, pero la situación internacional comenzó a cambiar también a consecuencia del impacto que tuvo el lanzamiento del Sputnik. En 1958, por iniciativa del investigador sueco Torsten Husén, un grupo de especialistas emprendió un gran esfuerzo internacional para desarrollar pruebas de rendimiento que pudieran aplicarse a los alumnos de sus respectivos países, para lo que debían basarse en los elementos comunes de los currículos de todos los sistemas educativos involucrados. 
Ese fue el origen de la International Association for the Evaluation of Educational Achievement (IEA), cuyos primeros estudios, sobre matemáticas y ciencias, se hicieron en las décadas de 1960 y 1970. En las de 1980 y 1990 iniciaron los estudios más conocidos, sobre matemáticas y ciencias (Third International Mathematics \& Science Study, TIMSS) y sobre lectura (Progress in International Reading Literacy Study, PIRLS), que después adoptaron un ritmo regular de aplicación, cada cinco y cuatro años. La IEA ha hecho estudios sobre educación cívica y otros. (Postlethwaite, 1985; 1994; Husén y Postlethwaite, 1996).

En muchos países, sin embargo, y en particular en la gran mayoría de los de América Latina, las pruebas de la IEA fueron casi desconocidas, y su impacto no fue significativo. Muy diferente fue lo que pasó con las pruebas promovidas por la Organización para la Cooperación y el Desarrollo Económico (OCDE), conocidas por la sigla PISA (Programme for Institutional Student Assessment, que se aplican cada tres años, desde el 2000.

Los resultados de PISA tienen una visibilidad creciente, en el también creciente número de países que participa en las sucesivas aplicaciones. Su difusión se ha vuelto un momento estelar en lo que se refiere al interés público por la calidad de la educación, de manera que son noticia de primera plana en la prensa escrita y en los medios audiovisuales. Tienen también un impacto considerable aunque desigual y muchas veces poco sustentado, como se verá más adelante- en las políticas de los sistemas educativos participantes. En muchos países esa difusión representa una fuerte presión para las autoridades educativas, que se ven orilladas a tomar decisiones importantes en forma apresurada y poco consistente, como reacción ante lo que, según los medios, es una situación catastrófica.

Un indicio del impacto mediático de los resultados de las pruebas de la OCDE: en Alemania, Noruega y otros países se utiliza la expresión PIS A shock para referirse a ello (Taut y Palacios, 2016: 12, 14). En una obra sobre los resultados de PISA 2006 (Roca y Martínez Rizo, 2009) se muestra que algo similar ha ocurrido en España y los países participantes en PISA de América Latina.

\section{Interpretación superficial y uso inadecuado de los resultados de PISA}

Los informes de cada aplicación de PISA se plasman en volúmenes con complejos análisis, pero los medios de comunicación destacan solamente los rankings, los ordenamientos de países basados en la media de puntos obtenidos por los jóvenes de la muestra evaluada, en competencia lectora, matemática y científica.

Eso es casi lo único que la mayoría del público conoce, y pocos van más allá, incluyendo a buena parte de las autoridades educativas nacionales y locales, y algunos de los especialistas que se interesan en el tema. La información que dan los ordenamientos, sin embargo, es limitada; se reduce a que cierto país está mejor o peor que tal otro, sin indagar en qué aspectos está la diferencia, si es o no significativa, si se modifica o no en el tiempo y, sobre todo, a qué se debe o, al menos, con que factores de la escuela y el entorno está asociada, aunque los informes contienen información sobre todos estos aspectos.

Otro uso de los ordenamientos se refiere al diseño de políticas para un sistema educativo nacional tratando de imitar lo que se hace en los países con mejores resultados. Además de las diferencias de los respectivos contextos demográficos, económicos, sociales y culturales, que hacen inviable transferir simplemente políticas que pueden haber funcionado en un país concreto, no se suele advertir que para que esa imitación tuviera sustento no basta identificar las llamadas "buenas prácticas" de los países ubicados en los primeros lugares de PISA, sino que habría que demostrar que son la causa de los mejores resultados de un país.

En muchos casos la forma en que los resultados de PISA se usan para orientar la política educativa se reduce, al parecer, simplemente a imitar políticas aplicadas en los sistemas que tienen los mejores resultados, lo que se espera debería bastar para mejorar los niveles de aprendizaje de los estudiantes del país en cuestión, y los resultados posteriores de PISA podrían corroborar. (Taut y Palacios, 2016:11, con base en Baird et al., 2011; Breakspear, 2012 y OECD, 2008) 
Un especialista en educación comparada dice que, aunque no es absurdo pensar que un país de resultados bajos en PISA puede aprender de otro de resultados altos, el problema es la forma en que se estudian otros sistemas educativos, y la forma en que se traducen a políticas los hallazgos:

Las reacciones de política suelen presentar una o más de estos tres rasgos: (i) extrema selectividad en la evidencia citada; (ii) una fe que parece fuera de lugar en la capacidad de las intervenciones de alcance nacional para transformar las prácticas de aula y, por ende, para elevar los estándares; (iii) una preferencia por intervenciones draconianas, de alto impacto [...] con base en la idea de que una vez que se identifica públicamente un problema $[\ldots]$ solamente las medidas más firmes del gobierno conseguirán impresionar al electorado (Alexander, 2012: 4).

Alexander dice que, en educación, como en economía, hay que tener cuidado para hacer atribuciones causales, y añade que los gobiernos parecen "creer ingenuamente que subir puntajes en pruebas hará mejorar la economía, y que copiar las políticas educativas de las naciones exitosas conseguirá a la vez subir los puntajes y sacarnos de la recesión” (2012: 5).

Establecer una relación causal entre dos variables, en efecto, no puede hacerse con base solo en una correlación, sino que implica controlar las otras variables que pueden incidir en la que se busca explicar, lo que no es fácil. Testigo de ello es la cantidad de estudios que tratan de explicar las diferencias de resultados escolares de distintos grupos de alumnos con numerosas variables, tanto de la escuela como del medio familiar y social en que viven los chicos.

Este tipo de estudios se multiplicó a partir de la difusión del estudio conocido como el Informe Coleman, en julio de 1966. Con datos de una enorme muestra de más de 600,000 alumnos, y utilizando análisis de regresión múltiple, que entonces eran avanzados, aunque adolecían de importantes limitaciones que luego se pusieron en evidencia, el Informe llegaba a la conclusión de que solo una pequeña fracción $(\sim 5 \%)$ de la varianza de los puntajes de los alumnos en las pruebas que se les aplicaron era predicha por las variables de la escuela, en tanto que el resto lo era por factores del entorno. (Coleman et al., 1966)

Este trabajo y otros de aquella época (v. gr. Bourdieu y Passeron, 1970; Bernstein, 1961) difundieron la idea, que todavía prevalece entre muchos investigadores educativos, de que la escuela, lejos de ser el mecanismo privilegiado de movilidad social, como se pensaba desde la Ilustración, era en realidad un mecanismo por el que las desigualdades sociales de una generación se transmiten a la siguiente, como proponía la teoría de la reproducción.

En las décadas siguientes, como se verá más adelante, otros estudios exploraron la cuestión, incluyendo los de la corriente de estudios de escuelas eficaces. Un número de la revista Sociology of Education, publicado para conmemorar 50 años del Informe Coleman, recoge lo que se sabe sobre el tema, mucho más matizado, y reconoce que la influencia de la escuela en los resultados de los estudiantes es importante, pero también compleja, con aspectos que promueven mayor igualdad, al lado de otros que favorecen la desigualdad. (Downey y Condron, 2016)

Un ejemplo notable de uso superficial de los resultados de PISA es el conocido Informe McKinsey, que analizó los sistemas educativos de 25 países, incluyendo a 10 de los de mejor situados en los ordenamientos basados en PISA.

Según el informe, aunque han invertido importantes recursos e implementado diversas reformas llenas de buenas intenciones, muchos sistemas educativos no han conseguido mejorar en décadas, mientras otros "tienen un desempeño cada vez mejor y alcanzan las mejoras más rápido". (Barber y Mourshed, 2007: 8-9).

El informe supone que los resultados en PISA bastan para identificar los mejores sistemas educativos, y en conclusión propone que los países de resultados bajos tomen medidas obvias que podría sugerir cualquier conocedor: 
- Que los mejores candidatos de cada generación escojan ser maestros pues la calidad de un sistema educativo tiene como techo la de sus docentes;

- Darles una formación que los prepare para ser docentes efectivos, porque la única manera de mejorar los resultados es mejorando la instrucción; y

- Que en cada escuela haya maestros bien preparados para que todo niño tenga una enseñanza de buena calidad, ya que la única forma de que el sistema tenga un alto desempeño es que todos los alumnos suban su nivel. (Barber y Mourshed, 2007: 17)

\section{Una noción más rica de la calidad educativa: el valor social de la educación}

Los sistemas educativos son parte esencial de las sociedades contemporáneas; en ellos se invierte una parte considerable de los recursos públicos, por lo que no pueden escapar a las exigencias de transparencia y rendición de cuentas. Por ello es importante contar con mediciones confiables de lo que saben los alumnos, lo que puede aportar elementos valiosos para sustentar decisiones de autoridades educativas y maestros. Pero hay que destacar que, si bien las competencias en lectura, matemáticas y ciencias son útiles para valorar el funcionamiento de los sistemas educativos, la calidad de estos comprende otras dimensiones.

La educación impacta el desarrollo económico de los países, en un mundo en que la competencia comercial es fuerte, y el peso de la ciencia y la tecnología cada vez mayor. Pero lo económico es sólo uno de varios aspectos importantes de la vida social. Tanto o más que alta productividad, un país necesita un sistema político que cuente con la confianza de la población por la responsabilidad y eficacia de legisladores, gobernantes y jueces; requiere también que los ciudadanos sean capaces de participar inteligentemente en la cosa pública, mediante los instrumentos de la democracia.

Los ciudadanos, además y sobre todo, tienen que estar bien preparados para los diversos papeles que desempeñarán durante su vida: de hijos y nietos; padres y abuelos; hermanos, amigos, enamorados y parejas; pero también consumidores de bienes materiales y simbólicos, lectores y televidentes, practicantes de un deporte o aficionados, conductores de autos o pasajeros, sanos o enfermos. Deben aprender a ganarse la vida, a disfrutarla, a transmitirla y a enfrentar con dignidad la muerte.

La escuela no es la única agencia de socialización de las nuevas generaciones, ni siquiera la más importante, pero sí tiene una parte de responsabilidad en esa tarea fundamental para el futuro de nuestras sociedades.

Desde la ilustración hasta mediados del siglo XX la postura prevaleciente destacaba la importancia de la escuela, que para sus más entusiastas defensores era el factor número uno del desarrollo social y económico. Luego algunos estudios -en especial el ya citado Informe Colemanpusieron en tela de juicio esa postura y, a fines de la década de 1960, llegó a prevalecer la opuesta, ejemplificada por la desescolarización de Ivan Illych: la escuela sería más bien un lastre para el desarrollo.

El avance de la investigación educativa la ha llevado a una postura más sensata, que reconoce el potencial de la escuela y sus límites. En este sentido apuntan trabajos en que los resultados de los alumnos se estudian a lo largo de varios años, aplicando pruebas de rendimiento al inicio y al fin de cada grado escolar.

Estos trabajos permiten hacer comparaciones más finas, que tienen en cuenta los resultados al principio y al fin de cada grado, con lo que se puede distinguir el avance durante el año escolar y el avance durante las vacaciones. La imagen que resulta de estas dos comparaciones es notable: durante el año escolar todos los niños muestran un avance similar, de modo que las diferencias que distinguían al inicio de un grado a los de nivel socioeconómico alto de los de nivel medio y bajo se mantienen iguales al fin del mismo. Durante los períodos vacacionales, en cambio, las brechas se agrandan: los chicos de nivel socioeconómico alto siguen avanzando como lo hicieron durante el ciclo escolar; los de nivel medio y, sobre todo, los de nivel bajo, avanzan menos, no avanzan o, incluso retroceden: al 
inicio del siguiente ciclo, después de las vacaciones, presentan resultados inferiores a los que tenían antes del verano, al fin del grado anterior. (Cfr. Entwistle, Alexander y Olson, 1997: 33-37)

Lo que estos resultados implican es que las brechas que separan a los alumnos acomodados de los más pobres, atribuibles inicialmente al hogar y al entorno social, no se agrandan debido a la escuela sino, precisamente, debido a su ausencia. Mientras los niños están bajo su influencia, la escuela consigue que todos avancen. Cuando no lo están, los niños privilegiados siguen aprendiendo, probablemente gracias a las actividades educativas a las que tienen acceso; los niños más pobres, en cambio, que no tienen esas facilidades, no aprenden, e incluso pierden algo de lo que habían conseguido aprender en el ciclo anterior.

Un argumento análogo, a contrario sensu, de la importancia de la escuela, lo dio el antropólogo y educador brasileño Darcy Ribeiro, en 1986, al analizar el aumento de la criminalidad juvenil, y relacionar el fenómeno con el hecho de no asistir a la escuela, o hacerlo en forma limitada.

Para seguir el argumento es necesario tener presente un rasgo del sistema educativo brasileño y otros países latinoamericanos en la segunda mitad del siglo XX: el llamado doble turno, que consistía en que en un mismo edificio escolar se atendía a unos grupos por la mañana y otros por la tarde, para poder dar cabida a todos los niños que cada año tocaban las puertas de la escuela en números cada vez mayores, como resultado de la explosión demográfica que hizo que la población se multiplicara por cuatro en 50 años.

En Brasil la presión demográfica obligó a que se establecieran hasta tres turnos en cada plantel, con solo tres horas y media teóricas de asistencia diaria por grupo. Al asumir la responsabilidad del sistema educativo de Río de Janeiro, tras la elección que marcó la vuelta a la democracia en ese país en 1984, Darcy Ribeiro señalaba las consecuencias de ese sistema para los niños de familias pobres, que no podían ir por la tarde a clases privadas de materias importantes, o llevar cursos extra de inglés, artes o deportes, como sus coetáneos más afortunados.

El severo diagnóstico -que hoy parece profético— que hizo el educador brasileño sobre las consecuencias de que la escuela brindara una atención tan limitada a los niños de las favelas de Río, y dejara que fueran educados principalmente por la calle y la pandilla, se plasma en frases que hoy tienen resonancias escalofriantes:

Nuestros niños están siendo preparados, con todo vigor, para la delincuencia. Se multiplican cada día las pandillas de niños abandonados... los que ya suman millares. ¿Qué sucederá mañana? No es imposible pensar que estas bandas deban ser combatidas por las fuerzas armadas cuando la policía confiese, finalmente, su fracaso para contenerlas. (Ribeiro, 1986b, pp. 22-23)

La actualidad del diagnóstico de Ribeiro se confirma ante la crisis de inseguridad por la que pasa un país como México, en el que la cifra de personas asesinadas por la delincuencia relacionada con el tráfico de drogas se sitúa en el orden de 2,000 mensuales en 2017, y las cifras serán rebasadas en 2018. Ya es demasiado tarde para evitar que sean preparados exitosamente para la delincuencia los niños mexicanos que fueron a la primaria en la última década del siglo pasado y no terminaron la secundaria obligatoria. Con sorpresa y terror lo estamos advirtiendo cuando el ejército ya tomó el lugar de la policía. También es tarde para conseguir que los jóvenes que desertan ahora en el tramo básico de la secundaria terminen la parte superior. Estamos sólo a tiempo de hacer algo para que los niños que comenzarán el recorrido escolar en los próximos años no deserten sin terminar al menos la secundaria obligatoria. Para ello hay que empezar fortaleciendo el sistema educativo desde la base, con preescolares y primarias de jornada completa y buena calidad para los niños más pobres, como comenzó a hacer Darcy Ribeiro en Río hace 25 años. (Ribeiro, 1986a)

Preciso que, a mi juicio, ser buenos ciudadanos no quiere decir ser súbditos obedientes, trabajadores dóciles o consumidores manipulables; quiere decir, en cambio, ser personas que respeten las normas de convivencia, pero que también sean capaces de cuestionarlas y sustituirlas por otras mejores, por medios democráticos, si se convencen de que no son las adecuadas. Nuestras 
democracias, en efecto, distan mucho de ser perfectas, aunque seguramente son bastante mejores que la mayor parte de las sociedades que las precedieron.

Las sociedades primitivas restringían la noción misma de ser humano a los miembros del propio grupo, en tanto que los de las tribus vecinas eran no-hombres hostiles que se podía matar y comer o esclavizar. En el seno de cada grupo regía la Ley del Talión. Después de milenios, en los orígenes de las tradiciones jurídicas que hoy aceptamos, los romanos definían lo justo como dar a cada quién lo que es suyo, entendían que el propietario de un bien tenía derecho de usar y abusar del mismo y que el concepto de bien incluía a los esclavos.

Poco a poco las nociones de justicia y derechos se fueron precisando: al final de la Edad Media y en la época de expansión europea del siglo XVI, con el ius gentium y la discusión sobre el derecho de conquista; en la Ilustración, con las ideas de democracia, ciudadanía, separación de iglesia y estado y de los poderes de este último, que plasmaron las revoluciones estadounidense y francesa; con las luchas sociales frente a la explotación del liberalismo manchesteriano y con los ideales del socialismo; con la acotación del egoísmo del bomo economicus en sociedades de bienestar de mediados del siglo XX; en las décadas recientes con la ampliación de las nociones de derechos humanos para incluir los sociales, los que tienen que ver con el medio ambiente y los que involucran a las generaciones siguientes.

Sin perder de vista que sigue habiendo -muchas veces con abrumadora presenciaimposiciones brutales y negociaciones mezquinas, importa destacar también que los alcances que hoy es normal atribuir a la noción de justicia incluyen tipos de solidaridad impensables hace no mucho tiempo. En el terreno educativo hoy es normal considerar que todos los niños tienen derecho a la educación elemental y media, y la obligatoriedad escolar se ha extendido ya en muchos países hasta los 18 años. No hace mucho el acceso a la educación se veía en forma muy distinta.

En la Francia ilustrada del XVIII La Chalotais sostenía: hay demasiados maestros, demasiadas escuelas... incluso el pueblo quiere estudiar... y criticaba a los hermanos de las escuelas cristianas por enseñar a leer y escribir a personas que sólo deberían aprender a dibujar y a manejar el cepillo y la lima pero ya no quieren hacerlo. El mismo Voltaire lo felicitó por ello y le escribió: Le agradezco que proscriba el estudio a los campesinos. Yo, que me dedico al cultivo de la tierra, lo que busco es tener mano de obra y no clérigos tonsurados... conviene que el pueblo sea conducido y no que sea instruido... me parece esencial que haya indigentes ignorantes... (Aries, 1973: 348)

La universalización de la educación elemental se aceptó desde mediados del siglo XIX, pero hubo que esperar un siglo más para que la idea de una escuela secundaria de tipo comprensivo comenzara a imponerse, siguiendo el camino que emprendieron desde el siglo XIX los Estados Unidos, y ahora todo el mundo trata de seguir, comenzando por Suecia, al terminar la Segunda Guerra Mundial.

Las actuales nociones de justicia y equidad son el resultado de complejos procesos, a través de los cuales los grupos humanos modifican la forma en que se perciben a sí mismos y a los demás. Este carácter de producto de una historia cultural se refleja en la vieja definición de la justicia como el mínimo de solidaridad que una sociedad considera exigible a sus miembros.

¿Y qué puede hacer la escuela en este sentido? Para responder esta pregunta retomo las palabras de Lorenzo Milani, el creador de la Escuela de Barbiana, cuyos alumnos lo hicieron conocido en los medios educativos de los años 1960 y 1970 con su Carta a una maestra de escuela.

En 1965, maestro y alumnos de Barbiana apoyaron públicamente a 31 objetores de conciencia, detenidos por negarse a cumplir el servicio militar. Milani fue acusado de apología de un delito. Enjuiciado por ello escribió su defensa en una carta abierta dirigida a los jueces en la que, apoyándose en la constitución italiana, sostenía el derecho a la objeción de conciencia como un avance de la ley en dirección de un mundo más cercano a la paz y más alejado de la guerra. Pero más allá de su impecable alegato jurídico, Milani defendía su postura como educador en una bella descripción de la dimensión valoral de esta tarea:

Voy a explicar ahora el problema de fondo de toda escuela. Así llegaremos, creo, a la clave de este proceso, porque a mí, maestro, se me acusa de haber defendido un delito. En otras palabras, se me acusa de haber hecho una escuela mala. Será necesario, pues, 
que nos pongamos de acuerdo sobre qué es una escuela buena. La escuela es distinta de un tribunal. Para ustedes, magistrados, sólo vale lo que es ley establecida. La escuela, en cambio, se sitúa entre el pasado y el futuro y debe tener presentes a ambos. La escuela es el difícil arte de conducir a los muchachos sobre un camino delgado como filo de navaja: por un lado, formarles en el sentido de la legalidad, y en esto se parece a la función del juez; por otro, formarles en la voluntad de mejorar las leyes, es decir, en sentido político y en esto se distingue de la función de los jueces.

Lo trágico del oficio de juez es que ustedes saben que deben juzgar con leyes que todavía no son del todo justas. Viven en Italia magistrados que en el pasado han tenido incluso que sentenciar a muerte a algunas personas. Y si hoy, que la pena de muerte ha sido ya abolida, todos nos horrorizamos por esas condenas, debemos agradecérselo a aquellos maestros que nos ayudaron a progresar enseñándonos a criticar la ley que antes estaba vigente. He aquí por qué, en cierto sentido, la escuela está fuera del ordenamiento jurídico. El muchacho no es todavía penalmente imputable y no ejerce aún derechos soberanos. Está apenas preparándose para ejercerlos el día de mañana y es, en este sentido, inferior a nosotros, pues debe obedecernos y nosotros respondemos por él. Pero en otro sentido el muchacho es superior a nosotros, porque mañana hará que se decreten leyes mejores que las que tenemos actualmente. Teniendo en cuenta esto, el maestro debe, en la medida en que le sea posible, ser profeta: debe escrutar los "signos de los tiempos", debe adivinar en los ojos de los jóvenes las cosas bellas que ellos verán claras mañana, y que nosotros hoy vemos sólo confusamente. (Milani, 1976)

El maestro debe reconocer que la insatisfacción de los jóvenes ante el mundo que les estamos legando muchas veces es justificada. Los problemas pueden abrumar y llevarlos a dos extremos perniciosos: rechazo violento, lleno de desesperación, amargura e impotencia; o aceptación cínica, acomodaticia y ventajista.

La escuela no es la única responsable de la formación de los futuros ciudadanos, y sus esfuerzos pueden ser insuficientes frente a los medios de comunicación y otros actores; pero la parte que le corresponde no es menor, en una sociedad en que las familias son cada vez menos autosuficientes, y la ausencia de escuela puede estar contribuyendo de manera no despreciable al deterioro que pone de manifiesto la violencia que castiga a países como México. La tarea de la escuela implica más que enseñar a leer y escribir $\mathrm{o}$, si se quiere, las competencias en lectura, matemáticas y ciencias. Evaluar la calidad de la escuela supone más que medir dichas competencias. Sin negar la importancia de que los instrumentos que se utilicen sean confiables, igualmente fundamental para una buena evaluación es precisar el referente adecuado para contrastar el resultado de la medición. En palabras de Milani, cuando afirmaba que para llegar a la clave del proceso al que estaba sometido, sería necesario ponerse de acuerdo sobre qué es una escuela buena, puesto que se le acusaba de haber hecho una escuela mala.

Lectura, escritura y aritmética: las tres erres del conocido dicho americano. Otro gran educador, Jerome Bruner, propone tres tareas igualmente importantes para la educación de nuestro tiempo, que propone sintetizar con tres palabras que comienzan con la letra pe. Según Bruner, la escuela hoy debe enseñar a sus alumnos a entender el pasado, el presente y lo posible. (1996: 86-99)

Bruner cuenta que en 1994 le pidieron cita dos altos funcionarios del sistema educativo ruso, lo que él atribuía al interés por sus estudios sobre enseñanza de las matemáticas. Pero no se trataba de eso; al tanto de los últimos trabajos del investigador, los rusos se interesaban por otro tema:

¿Qué podemos hacer - preguntaron- para enseñar la historia de Rusia en el último siglo, incluyendo 75 años de régimen comunista? ¿Simplemente como un enorme error? ¿Como Rusia manejada por un grupo de oportunistas desde el Kremlin? ¿O puede reconstruirse el pasado, no sólo para que adquiera sentido con todo y sus tragedias, sino 
también para que cobre sentido la forma como podría esbozarse el futuro? (Bruner, 1996: 88)

Toda sociedad necesita una visión del pasado, o sea cierta interpretación de su historia; una visión del presente, o sea cierto análisis de su problemática actual; y una visión del futuro, o sea una idea de lo que es posible, de lo que la sociedad podría ser, si todos sus miembros trabajaran para conseguirlo. La educación puede y debe contribuir a formar esas tres visiones; por ello me gusta la propuesta de las tres pes de Bruner: la escuela -en Rusia como en México o en España - tiene que enseñar el pasado, el presente y lo posible. Por eso pienso, con Milani, que la escuela debería desarrollar en los alumnos el sentido de respeto a las leyes y, a la vez, la capacidad de trascenderlas.

Creo que es la misma idea expresada por Fernando Savater, en el sentido de que los maestros debemos transmitir a nuestros alumnos lo que creemos mejor de lo que fuimos, pero que sabemos que les será insuficiente... como fue insuficiente también para nosotros. Debemos decirles: transfórmenlo todo, mejórenlo todo, empezando por ustedes mismos, pero guarden conciencia de qué es y cómo es, de qué fue y cómo fue lo que quieren transformar. (Savater, 1997: 193)

Una buena escuela, además de hacer que sus alumnos dominen la lectoescritura y la matemática, las ciencias naturales y la computación, será una que cumpla con su parte en el rol de cultivar el pensamiento utópico, en el sentido que emplea Javier Muguerza cuando, respondiendo a una pregunta sobre si tal pensamiento debería abstenerse de decir nada acerca del futuro, afirma:

[...] hay un sentido en el que la utopía, desde un punto de vista ético y no desde la perspectiva bastante menos defendible de una filosofía de la historia que se asigna como misión la prospección del futuro, tendría sin duda algo que ver con este último: aquel en el que Kant, tras de formularse la pregunta "¿Cómo es posible una historia a priori?”, se respondía a sí mismo "Cuando el adivino hace y dispone lo que anuncia", esto es, se convierte en actor. (Muguerza, 1990: 693-694)

Como un ejemplo de que un actor educativo puede ser relevante como actor de la historia en general, puede recordarse el trascendental juicio que hizo que el sistema educativo de los Estados Unidos rechazara el principio de segregación racial que lo regía desde la independencia del país, a fines del siglo XVIII.

En 1952, la familia de la niña afroamericana Linda Brown decidió demandar al Consejo de Educación del Condado de Topeka por prohibir que su hija asistiera a una escuela reservada a los niños blancos. La Suprema Corte de Justicia le dio la razón con la sentencia que inició la desegregación racial del sistema educativo americano, al definir como anticonstitucional obligar a los niños negros a asistir a escuelas distintas de las que atendían a los blancos, aún en el discutible caso de que la calidad de la enseñanza recibida en unas y otras fuera similar.

La Corte rechazó el principio de que era correcto educar a los niños en escuelas separadas pero iguales, según la expresión generalmente aceptada. Pero lo que conviene destacar es el argumento que esgrimió el abogado que defendía la postura segregacionista del Condado y la consideración en contrario de la Corte. El abogado del condado argumentaba que la demanda del papá de Linda Brown era un inútil intento por cambiar 300 años de actitudes tan arraigadas culturalmente que era imposible modificarlas. El juez Felix Frankfurter respondió:

En este mundo las actitudes no se cambian en abstracto, por ejemplo leyendo algo... Las actitudes son en parte el resultado del trabajo y de la acción... No hay que doblar las manos y esperar que cambien solas. (Friedman, 1969: 412-413, en Reeves, 2007: 248)

\section{Conclusión}

La preocupación por la calidad de la educación que se ha extendido en el mundo puede verse como positiva, en la medida en que ha conseguido que una parte de la sociedad, que no mostraba interés por estas cuestiones, se preocupe por lo que ocurre en la escuela y participe en acciones de 
mejora, a partir de la conciencia de que es importante para el futuro de los niños y de la sociedad. Algunas formas que adopta dicha preocupación, sin embargo, son dañinas para la educación, como la destructiva competencia que propician los engañosos ordenamientos derivados de las pruebas en gran escala y las superficiales discusiones derivadas de ellos, a las que son tan proclives los medios de comunicación.

Sin sobreestimar las posibilidades de la escuela, creo que algo podrá hacer mejor que la calle para reducir la violencia, si da una buena educación a lo largo de una jornada completa en la que, además de enseñar lengua, matemáticas y ciencias, se ofrezca a los niños, sobre todo a los más pobres, un ambiente respetuoso, seguro e incluyente, en el que más que nada aprendan los valores cívicos y de solidaridad, viviéndolos con sus compañeros y sus maestros.

Es importante estudiar si las escuelas son buenas, pero no con la visión miope de los rankings, sino a partir de la convicción de que no lo serán si no contribuyen a preparar jóvenes que quieran transformar su sociedad y mejorarla, y que también sepan perdonar a sus mayores por no haberlo conseguido, sabiendo que ellos tampoco lo conseguirán del todo.

\section{Referencias bibliográficas}

Aries, Ph. (1970). L'enfant et la vie familiale sous l'ancien régime. París, Seuil.

Alexander, R. (2012) Moral panic, miracle cures and educational policy: what can we really learn from international comparison? Scottish Educational Review 44 (1), 4-21.

Baird, J. A., Isaacs, T., Johnson, S., Stobart, G., Yu, G., Sprague, T. \& Daugherty, R. (2011). Policy effects of PISA. http://research-information.bristol.ac.uk/en/publications/policy-effects-ofpisa(833739c4-7e0a-4c18-b249-a3f12120065f).html

Baker, E. y Choppin, B. (1990). Minimum competency testing. En Walberg, H y Haertel, H. (Eds.). The International Encyclopedia of Educational Evaluation (pp. 499-502). Nueva York: Pergamon Press.

Barber, M. y Mourshed, M. (2007). How the World's Best-Performing School Systems Come Out on Top. McKinsey \& Co. (Cómo hicieron los sistemas educativos con mejor desempeño del mundo para alcanzar sus objetivos. PREAL, $\mathrm{N}^{\circ}$ 41, Julio 2008).

Bernstein, B. (1961). Social Class and Linguistic Development: A Theory of Social Learning. En Halsey, A. H., Floud, J., y Anderson, C. A. (Eds.). Education, Economy \& Society. A Reader in the Sociology of Education (pp. 288-314). New York: The Free Press of Glencoe.

Bourdieu, P. y Passeron, J. C. (1970). La reproduction. Élements pour une théorie du système d'enseignement. Paris: Minuit.

Breakspear, S. (2012). The policy impact of PISA: An Exploration of the Normative Effects of International Benchmarking in School System Performance. OECD Journals, 71, 1-32. http://doi.org/10.1787/19939019

Bruner, J. The Culture of Education. Cambridge. Harvard University Press, 1996.

Coleman, J. S., Campbell, E. Q., Hobson, C. J., McPartland, J., Mood, A. M., Weinfeld, F. D., y York, R. L. (1966). Equality of Educational Opportunity. Washington: Office of Education.

De Landsheere, G. (1996). La investigación educativa en el mundo. México: Fondo de Cultura Económica [Edición original en francés de 1986].

Downey, D. B. y Condron, D. J. (2016). Fifty Years since the Coleman Report: Rethinking the Relationship between Schools and Inequality. Sociology of Education, 89 (3), 207-220.

Entwistle, Doris R., K. Alexander y L. S. Olson (1997). Children, Schools and Inequality. Boulder. Westview Press.

Husén, T. y Postlethwaite, T. S. N. (1996). A brief history of the International Association for the Evaluation of Educational Achievement (IEA). Assessment in Education: Principles, Policy \& Practice, 3(2), 129-141.

Martínez-Arias, R. (1995). Psicometría: teoría de los tests psicológicos y educativos. Madrid: Síntesis. 
Mathison, S. y Ross, E. (2008). The Nature and Limits of Standards-Based Reform and Assessment. Nueva York: Teachers College Press.

Milani, Lorenzo (1976). Carta a los jueces. Sinite, 49 (enero-marzo), 111-134.

Muguerza, Javier. Desde la perplejidad. Madrid, Fondo de Cultura Económica, 1990.

Postlethwaite, T. S. N. (1985). International Association for the Evaluation of Educational Achievement. En Husén, T. y Postlethwaite, T. S. N. (Eds.). The International Encyclopedia of Education (pp. 2645-2646). Nueva York: Elsevier.

Reeves, Douglas (2007). Challeges and Choices: The Role of Educational Leaders in Effective Assessment. En Reeves, Douglas, ed. Ahead of the Curve. The Power of Assessment to Transform Teaching and Learning (pp. 227-251). Bloomington, Solution Tree Press.

Ribeiro, Darcy (1986a). O livro dos CIEPs. Rio de Janeiro, Bloch Editores.

Ribeiro, Darcy (1986b). Revolución en la educación. El sistema escolar brasileño. Nueva Sociedad, 86 (noviembre-diciembre), 22-32.

Roca, E. y Martínez Rizo, F.(Coords.) (2009). Iberoamérica en PIS A 2006.Madrid. Santillana.

Taut, S., y Palacios, D. (2016). Interpretaciones no intencionadas e intencionadas y usos de los resultados de PISA: Una perspectiva de validez consecuencial. Revista electrónica de Investigación y Evaluación Educativa RELIEVE, 22(1), art. M8.

Savater, Fernando. El valor de educar. México. Instituto de Estudios Educativos y Sindicales de América, 1997.

\section{Para citar este artículo}

Martínez-Rizo, F. (2018). La preocupación por la calidad de la educación y su valor social. Revista Fuentes, 20(2), 17-27. [Fecha de consulta: dd/mm/aa]. doi: http://dx.doi.org/10.12795/revistafuentes.2018.v20.i2.01 